\title{
La indexación de las revistas de psicología de Colombia en las bases de datos científicas españolas: un estudio exploratorio*
} Indexing of Colombian psychology journals in Spanish scientific databases: an exploratory study

\author{
Pedro VÁzquez-Miraz \\ Universidad Tecnológica de Bolívar, Colombia \\ ORCID: https://orcid.org/0000-0002-5801-1728
}

a Autor de correspondencia. Correo electrónico: pvasquez@utb.edu.co

Para citar este artículo: Vázquez-Miraz, P. (2021). La indexación de las revistas de psicología de Colombia en las bases de datos científicas españolas: un estudio exploratorio. Universitas Psychologica, 20, 1-13. https://doi.org/10.11144/Javeriana.upsy20.irpc

\section{RESUMEN}

En el siguiente documento se presenta un trabajo exploratorio en el que se analiza la relevancia de las principales revistas de psicología colombianas, aquellas que están indexadas en los indicadores nacionales (Publindex) e internacionales (Web of Science y Scopus) más importantes, en función de las cifras aportadas por dos bases de datos científicas de origen español (CIRC y MIAR) de amplio uso en el ámbito bibliométrico. Por medio de un análisis correlacional de Spearman entre estos tres elementos y el índice H5 de Google Académico, los resultados muestran la existencia de una fuerte correlación entre Publindex y el índice H5 $(r=0.86 ; p$ $=0.001$ ) no habiendo diferencias significativas entre Publindex y las matrices europeas. Además, se puede considerar que la matriz de datos CIRC subestima a este tipo de publicaciones en comparación con los otros tres anteriores medidores y, por ende, no recomendaríamos a los psicólogos colombianos el uso de esta última base de datos española.

Palabras clave

psicología; publicaciones científicas; indicadores bibliométricos; indicadores bibliográficos; indexación de revistas.

\begin{abstract}
An exploratory work that analyzes the relevance of the principal Colombian psychology journals [those that are indexed in the most important national (Publindex) and international (Web of Science and Scopus) indicators], depending on the figures provided by two Spanish scientific databases (CIRC and MIAR), widely used in the bibliometric field is presented in the following document. By means of a Spearman correlation analysis between these three elements and Google Scholar $\mathrm{H} 5$ index the results show the existence of a strong correlation between Publindex and H5 index $(r=0.86 / p=0.001)$ no there being significant differences between Publindex and the European matrices. In addition, it can be considered that CIRC data matrix underestimates this type of publications in comparison with the other three meters and therefore we would not recommend the use of this last Spanish database to Colombian psychologists.

Keywords

psychology; scientific publications; bibliometric indicators; bibliographic indicators; journal indexing.
\end{abstract}


La divulgación del conocimiento tiene tanta historia como la propia ciencia y esta siempre fue una pieza esencial que acompañó los acontecimientos sociales, culturales, políticos, militares, económicos y tecnológicos más importantes, pues como afirman Ruiz-Corbella et al. (2014), la necesidad de transmitir a la comunidad académica los avances que se encuentran y/o logran ha sido una constante a lo largo de toda la historia de la humanidad. De esta situación no sería la excepción el ámbito de la psicología en Colombia, pues los investigadores de esta área del conocimiento científico también quieren que sus descubrimientos sean divulgados en los medios de mayor prestigio y difusión posibles (ya que su rendimiento laboral es medido por estas variables), siendo estos dos conceptos muchas veces definidos por multinacionales con ánimo de lucro (Ordorika, 2018).

Es más que obvio que el papel fundamental y básico para lograr la transmisión de información fue la escritura (López \& Yung, 1998; MederosMartin \& Ruiz-Cabrero, 2001). Los papiros egipcios, las tablillas asirias, las bibliotecas de Pérgamo y Alejandría de la época antigua o los monasterios europeos de la Edad Media fueron todos ellos elementos que almacenaban el saber humano (Fernández-Abad, 2006). Para Mendoza y Paravic (2006), son tres los momentos clave en los que la ciencia tradicional fue evolucionando y transformándose en lo que se podría denominar actualmente como ciencia moderna: los trabajos de Copérnico (siglo $\mathrm{XVI}$ ), las investigaciones y planteamientos de Kepler, Descartes y Newton (siglo XVII) y las necesidades de discernimiento posteriores que ya necesitaba un mundo, incipientemente global, en entornos tan dispares como la navegación, el comercio o la agricultura.

Los antecedentes más claros del origen de las revistas científicas modernas, tal como hoy las conocemos, se pueden situar en la Europa del siglo XVII, época en la que se fundaron la Royal Society (Inglaterra) y la Académie Royale des Sciences (Francia) cuya finalidad era difundir y compartir los hallazgos realizados por sus miembros, siendo las primeras publicaciones periódicas dónde se plasmaban los avances en estas instituciones el Philosophical Transactions of the Royal Society y el Journal de sçavans (Mendoza \& Paravic, 2006; Ruiz-Corbella et al., 2014). La proliferación y globalización de este tipo de literatura desde finales del siglo XIX, como afirman Rueda-Clausen et al. (2005), ha creado la obligación de elaborar un sistema de evaluaciones tales como escalas e indicadores en el que se pueda calificar este tipo de producciones científicas. El primer trabajo bibliométrico en el que se comparan textos académicos de diferentes países fue el elaborado por Alphonse de Condolle en 1885 titulado Historie des sciences et des savants depuis deux siècles (Rueda-Clausen et al., 2005).

Ya es en pleno siglo XX, cuando la comunidad investigadora asume que el recuento de citaciones realizadas de un determinado artículo científico es un elemento comparativo de la calidad de este documento y también un indicador del impacto que tiene ese producto en el medio en el que fue publicado. Del mismo modo el concepto de factor de impacto, una noción matemática calculada en función del cociente del número de citaciones de una revista al año por la cantidad de artículos que se publicaron en ese periodo (Espinosa \& Insuasty, 2015), se aplicaría a las propias cualidades de las revistas científicas. El siglo pasado terminaría con la aparición de la célebre plataforma de información (accesible por Internet) JCR (Journal Citation Reports), base de datos que recopila y analiza datos bibliográficos de las revistas más autorizadas en las áreas de las ciencias (Rueda-Clausen et al., 2005).

En el siglo XXI, la actividad investigativa en las últimas décadas ha sufrido una completa revolución respecto a la metodología tradicional empleada hasta finales del siglo pasado debido, en gran parte, al dominio absoluto de las tecnologías de la información y la comunicación (TIC) que impera en los tiempos presentes. Así pues, la bibliometría, disciplina integrada en las ciencias métricas que permite analizar la producción científica, está en continua evolución integrando siempre en su seno las nuevas tecnologías y de la comunicación (López, 2007) por lo que la búsqueda de mejoras en un índice de medición 
o la creación de uno nuevo es una constante (Pérez-Anaya, 2017). Este cambio es más que evidente en el campo de la producción y lectura de información científica pues el formato digital ha revolucionado la demanda de este tipo de conocimiento. Si en el año 2005 el $20 \%$ de los artículos consultados estaban en formato electrónico, en el año 2014 estos ya eran más de la mitad (Torres-Salinas et al., 2014).

Este avance hacia el formato electrónico sigue imparable tanto en la formación (Díaz et al., 2017) como en la edición de nuevos productos (Mas-Bleda \& Aguillo, 2015). Si en el pasado, la búsqueda de información en bases de datos, debido a su escasa disponibilidad al depender el investigador en exclusiva de soportes físicos, era una de las mayores dificultades, la vigente revolución tecnológica ha facilitado esta labor académica de forma considerable al permitir Internet el fácil acceso a una infinita cantidad de documentación en formato digital, la cual puede ser de libre disposición (Tabla 1).

\section{Tabla 1}

Tamaño de las principales bases de datos bibliográficas

\begin{tabular}{lr}
\hline \multicolumn{1}{c}{ Base de datos } & Número de documentos \\
\hline Google Scholar & 170000000 \\
Web of Science & 167127889 \\
ResearchGate & 81000000 \\
Microsoft Academic Search & 80000000 \\
Web of Science Core Collection & 61856513 \\
Scopus & 61583942 \\
Mendeley & 32000000 \\
Academia.edu & 10767769 \\
\hline
\end{tabular}

Fuente: Orduña-Malea et al. (2016)

Así pues, el advenimiento de esta sociedad de la información hizo que el tema de la producción y propagación de la ciencia haya instaurado hoy en día a las revistas científicas como el medio académico por excelencia de difusión de ideas, teniendo este tipo de publicaciones una serie de objetivos básicos por desarrollar (VillalobosGalvis \& Puertas-Campanario, 2007):

Transformarse en vehículos de transmisión del conocimiento de nuevos hallazgos definiendo y difundiendo el vocabulario científico.
Ser una zona destacada para la discusión y aceptación del conocimiento y convertirse en herramientas de enseñanza.

Favorecer la divulgación de la ciencia y promocionar el desarrollo científico.

Convertirse en indicadores científicos nacionales y regionales y obtener el reconocimiento de la contribución de un investigador.

\section{Marco teórico}

El origen de las revistas científicas colombianas tiene una larga tradición histórica, pudiéndose afirmar que las primeras ediciones de esta índole surgen en diferentes épocas del siglo XX (Salas et al., 2018). La primera impresión científica colombiana del medio psicológico (que se pueda definir como tal) fue la Revista de Psicología [fundada en 1956 por Mateo Mankeliunas en la Universidad Nacional de Colombia (Ossa \& Cudina, 2016)], a la que en años posteriores se les unirían otras revistas de gran trascendencia e importancia como la Revista Latinoamericana de Psicología (Salas et al., 2018). Lamentablemente, muchas de ellas, creadas durante el período 1979-1986, desaparecieron rápidamente y contribuyeron con su aparición a una competición cainita en la que se dispersaron esfuerzos y recursos (Jarama-Barrios et al., 2012). A pesar de las carencias económicas de la zona, en la actualidad es en los países iberoamericanos donde los boletines especializados de psicología alcanzan una mayor relevancia social y educativa, al ser esta la región del mundo que cuenta con el mayor número de psicólogos (López-López, 2018).

Estos medios regionales (de acceso abierto), entendidos como toda publicación editada en Colombia, ranqueada en las bases de datos Scopus o la Web of Science y cuya misión primordial obedece a la de dar a conocer investigaciones en el campo de la psicología, serían la excepción a la regla establecida basada en el enorme poder de las grandes editoriales privadas cuya información es exclusiva mediante pago económico (Elsevier, dueña de Scopus o 
Clarivate Analytics, propietaria de WoS): otra de las dificultades de los investigadores de hoy en día (y más en particular los de Colombia) a la hora de realizar su trabajo debido a la inaccesibilidad de datos por la limitación presupuestaria (Ordorika, 2018). Propuestas como el movimiento del Plan $\mathrm{S}$ en contra de la hegemonía de los sistemas de indexación privada a favor de las revistas de open access (Schiltz, 2018) son una alternativa muy interesante que cada vez tiene una mayor incidencia en América Latina. Guste o no, el enorme progreso tecnológico hizo que bases de datos privadas como Scopus y Web of Science sean las herramientas dominantes (VillegasGallego, 2013) y tengan una gran aceptación en la comunidad de psicólogos colombianos a pesar de que estas presenten un sesgo a favor de lo anglosajón, el idioma inglés y a las temáticas técnicas (Delgado-López et al., 2014; Moed, 2005; Ordorika, 2018; Osca-Lluch et al., 2013).

En relación con el aspecto lingüístico, el hábito de muchos estudiosos y publicaciones de no usar como lengua de trabajo el inglés (la lengua internacional por excelencia) hizo que países latinos realizaran esfuerzos comunes a la hora de promover la visibilidad de las revistas latinoamericanas, españolas y portuguesas (Villalobos-Galvis \& PuertasCampanario, 2007). Esta preocupación llevó a la creación de sistemas de información de revistas específicos como LATINDEX (Sistema regional de información en línea para revistas científicas de América Latina, el Caribe, España y Portugal) o Scielo (Scientific Electronic Library Online).

La situación descrita es la que justifica el presente estudio al asumirse que para los psicólogos colombianos es fundamental conocer cómo varios indicadores bibliográficos de habla hispana de gran relevancia [de Colombia (Publindex) y de España (CIRC y MIAR)] indexan las principales revistas donde este gremio suele visualizar sus trabajos de mayor calidad (las revistas colombianas de psicología). De este modo, el propósito básico de esta investigación es identificar si existen diferencias significativas entre estos indicadores bibliográficos respecto a este tipo de revistas. Un aspecto inédito que apenas fue estudiado en la literatura científica relacionada con la bibliometría y/o la psicología.

La notoriedad pública de una revista es un factor elemental que permite conquistar una mayor visibilidad de los trabajos. Por ello, es de un gran valor que el documento se encuentre, de manera fundamental, catalogado e indexado en la mayor cantidad de bases de datos especializadas y que alcance el mayor reconocimiento posible como componente de alto prestigio y calidad. Así, el análisis de la publicación de artículos es la unidad estándar de medida de la calidad de la mayoría de los procesos investigativos (Masip, 2011), y también lo es en Colombia. Como indican Rueda-Clausen et al. (2005) las revistas científicas poseen en la actualidad un rol protagónico en la evaluación de la actividad científica de los investigadores y sus respectivos grupos al ser calificados en base al impacto de las publicaciones donde se exhiben sus hallazgos.

\section{La catalogación de revistas en Publindex}

En Colombia, el organismo encargado de la investigación y su promoción (dependiente del Departamento Administrativo de Ciencia, Tecnología e Innovación) es Minciencias [el antiguo Colciencias (Salazar et al., 2013), entidad creada en 1968 por medio del Decreto Nacional 2869 (Valencia et al., 2017)], institución que realiza el servicio de indexación de las revistas por medio de Publindex, un índice, creado a finales del siglo XX, cuyo último objetivo es medir la calidad científica de la investigación colombiana., su accesibilidad y su visibilidad (Rodríguez et al., 2015).

Con la creación del Índice Bibliográfico Nacional (IBN Publindex), se pretendía incentivar la publicación de nuevas obras y conseguir una significativa transformación en las revistas colombianas de la época (escasas en número, con baja visibilidad y predominantemente ligadas al espacio religioso), pasándose de 29 revistas en el año 1996 a 126 (año 2014) y 541 en el 2014 (Valencia et al., 2017). A finales del siglo XX, el gobierno 
colombiano emite una ley que vincula un aumento salarial a los profesores e investigadores de las universidades públicas con su producción científica. Esto condujo a la formación de normativas para cuantificar estos incrementos monetarios en base al tipo y nivel de calidad de los productos que se presentaran (Charum, 2004). La disparidad y laxitud con la que se interpretaba la legislación por parte de las instituciones universitarias públicas de Colombia provocó que, en el año 2002, se enfatizara, con la promulgación de una nueva ley, la importancia de manejar adecuadamente una calificación que evaluara productos de investigación y las propias revistas especializadas (colombianas y extranjeras) en función de su calidad (Charum, 2004).

Apareció, con este fin, el Sistema Nacional de Indexación y Homologación de Revistas Especializadas de Ciencia, Tecnología e Innovación de Colombia (Publindex), el medio desde el que se definen los criterios de calidad de las revistas científicas, y por los que se rige su clasificación. Este índice está formado por cuatro categorías (cuartiles) de orden creciente de prestigio y calidad: C [4], B [3], A2 [2] y A1 [1]. Para poder entrar en esta medición, una revista debe publicar un número determinado de artículos de forma constante y los trabajos publicados deben ser resultado de investigaciones, los cuales están presentados en el formato exigido por la publicación editora, siendo recomendable que la revista forme parte de índices internacionales (Espinosa \& Insuasty, 2015).

Publindex, en resumen, es la herramienta central a la que los investigadores colombianos se dirigen para comparar sus trabajos, y conocer la importancia y relevancia de su producción en su entorno más cercano. Un instrumento que no está exento de críticas, pues, aunque varias de sus reformas han creado un índice más internacional, más riguroso y selectivo en aras de cosechar una mayor excelencia investigativa (Díaz, 2017; Gómez-Marín \& Palacios, 2018; Villegas-Gallego, 2013), sus críticos afirman que también se ha fomentado la endogamia y se ha penalizado de manera injusta a la mitad de las revistas de este país andino (López-López, 2017).

\section{La catalogación de revistas en CIRC}

La Clasificación Integrada de Revistas Científicas (CIRC) es un instrumento de medida español que valora publicaciones del ámbito de las ciencias con base en aspectos bibliométricos que determinan su calidad, los cuales fueron recogidos previamente por diferentes agencias hispanas como la CNEAI (Comisión Nacional Evaluadora de la Actividad Investigadora) o la ANECA (Agencia Nacional de Evaluación de la Calidad y Acreditación) (Torres-Salinas et al., 2010). La clasificación de CIRC está formada por cuatro categorías de orden creciente de prestigio y calidad: D [5], C [4], B [3] y A [2]. Existe también una categoría de excelencia $(\mathrm{A}+)$ [1] en la que se recogen las revistas de mayor impacto y prestigio internacional, las auténticas publicaciones referentes de sus respectivas disciplinas.

Este índice fue presentado el año 2011 por especialistas de la Universidad de Navarra, el Instituto de Estudios Documentales sobre Ciencia y Tecnología, la Universidad Carlos III de Madrid y la Universidad de Granada (Serrate \& Rovira, 2017), siendo el núcleo originario de esta ordenación la empresa EC3metrics, una spin-off empresarial de esta última institución educativa, para solucionar la falta de indicadores bibliométricos para el estudio de la actividad en las ciencias sociales (Torres-Salinas et al., 2010).

En la actualidad, el CIRC y sus posteriores actualizaciones son una herramienta para evaluadores e investigadores que ha sobrepasado su espacio original (el campo de las humanidades y ciencias sociales de España) debido a su alta capacidad recopiladora. Recordemos que el objetivo último de esta clasificación es generar un instrumento de medida bibliométrico que facilite el realizar comparaciones y el compartir información entre investigadores, al basarse este elemento en las citaciones de las revistas y el acatamiento en los patrones de calidad editorial (Torres-Salinas et al., 2010). 


\section{La catalogación de revistas en MIAR}

La Matriu d'Informació per l'Avaluació de Revistes (MIAR) es un sistema hispano de gran aceptación por los investigadores de esta nación europea, basado inicialmente el Sistema de Evaluación de la Investigación en Humanidades y Ciencias Sociales (1997-2000) de la Generalidad de Cataluña (Rodríguez-Gairín et al., 2011), sistema que fue modificado por la Facultad de Biblioteconomía y Documentación de la Universidad de Barcelona al subsanar los errores, incoherencias y fallas detectados en este anterior catálogo (Urbano, 2003).

MIAR se podría definir como un directorio de libre acceso actualizado que mantiene un histórico y que permite consultar la evolución de un indicador de años anteriores (SomozaFernández et al., 2017). Su objetivo principal es el de cuantificar la indización en bases de datos de revistas de humanidades y ciencias sociales (Rodríguez-Gairín et al., 2011), y así cubrir el déficit de cobertura de citas internacionales en esta área del conocimiento en la Web of Science (Rodríguez-Gairín et al., 2011), una base de datos que no está exenta de la existencia de revistas depredadoras (Somoza-Fernández et al., 2016).

Esta matriz de datos se actualiza anualmente; reúne datos clave para la evaluación de revistas por medio de la correspondencia entre revistas, bases de datos, repertorios y catálogos de bibliotecas, siendo por consiguiente MIAR una herramienta de apoyo para identificar y evaluar la difusión de los textos científicos (RodríguezGairín et al., 2011).

\section{Metodología}

Por medio de un estudio bibliométrico de carácter descriptivo, se han analizado, de forma exclusiva, los indicadores de Publindex, CIRC, MIAR y H5 de Google de las revistas colombianas de psicología que estaban catalogadas en la Web of Science, Scopus y en la propia Publindex a finales del año 2018 (siendo en total catorce revistas), haciéndose las pertinentes comparaciones entre los mencionados índices. En este trabajo, no fueron revisadas las publicaciones de salud mental, psiquiatría o ciencias sociales de corte generalista y a diferencia de Ávila-Toscano y Marenco-Escuderos (2016), la revista Cultura, Educación y Sociedad no ha sido considerada específica del ámbito de la psicología. Esta tarea de delimitación conceptual no fue algo sencillo, pues como afirman Jarama-Barrios et al. (2012), estudiar por medios bibliométricos una comunidad como la de psicología en Colombia tiene como principal dificultad el carecer de información exhaustiva sobre su producción científica.

Para hacer una comparación neutral entre las clasificaciones colombianas y españolas, también se ha recogido un parámetro muy utilizado procedente de Estados Unidos: el índice $\mathrm{H}$, un indicador propuesto por Hirsch (2005) que permite comparar la productividad científica de los investigadores de forma individual y la calidad de las revistas. Un elemento de evaluación usado a través de herramientas muy populares como Google Scholar (Silva-Ayçaguer, 2012), el cual fue comparado con Scopus y la WoS en diversos estudios (Bar-Ilan, 2008; Martín-Martín et al., 2018).

Una vez clasificadas las diferentes revistas de psicología en función de los criterios propios de Publindex, CIRC, MIAR y H5 de Google, se calcularon los respectivos cuartiles (por medio del programa estadístico SPSS, v.23) de las puntuaciones MIAR y H5 de Google, para finalmente calcular las correlaciones correspondientes con las anteriores cifras y las clasificaciones de Publindex y CIRC. Aunque el escalafón de CIRC está formado por cinco niveles, ninguna revista colombiana de psicología estuvo situada en la posición más elevada de esta clasificación durante el estadio temporal de medición analizado.

\section{Resultados}

A finales del año 2018, se han evaluado a catorce revistas colombianas de psicología (el resto no estarían valoradas ni en Publindex, CIRC ni MIAR) introduciendo el escalonamiento 
numérico de Publindex, CIRC y las puntuaciones en cuartiles de MIAR y H5 de Google (Tabla 2). Los principales datos son los siguientes:

Ninguna revista en categoría A1.

Una revista en categoría A2 (Revista Latinoamericana de Psicología). Esta publicación indexada está en Scopus y en la Web of Science (Social Science Citation Index).

Ocho revistas en categoría B. Una obra está indexada en WoS y Scopus (Universitas Psychologica) y otra se encuentra en Scopus (Acta Colombiana de Psicología).

Cuatro revistas en categoría C.

\section{Tabla 2}

Evaluación de revistas colombianas de psicología (año 2018)

\begin{tabular}{lrrr}
\hline \multirow{2}{*}{ Nombre de la Revista } & \multicolumn{3}{c}{ Categorización } \\
\cline { 2 - 5 } & Publindex CIRC & MIAR & H5 Google \\
\hline Revista Latinoamericana de Psicología & A2 [2] A [2] & $11[1]$ & $19[1]$ \\
Acta Colombiana de Psicología & B [3] C [4] & $9.8[3]$ & $10[3]$ \\
Diversitas: Perspectivas en Psicología & B [3] D [5] & - & $10[3]$ \\
Pensamiento Psicológico & B [3] D [5] & $6.1[4]$ & $10[3]$ \\
Psicología desde el Caribe & B [3] D [5] & $6.5[3]$ & $12[2]$ \\
Psychologia: Avances de la disciplina & B [3] D [5] & - & $11[2]$ \\
Revista CES Psicología & B [3] C [4] & - & - \\
Suma Psicológica & B [3] D [5] & $9.9[2]$ & $10[3]$ \\
Universitas Psychologica & B [3] B [3] & $10.7[1]$ & $21[1]$ \\
Informes Psicológicos & C [4] D [5] & $6.1[4]$ & $4[4]$ \\
Pensando Psicología & C [4] C [4] & $9.6[3]$ & - \\
Psicogente & C [4] C [4] & $6.3[4]$ & $5[4]$ \\
Revista Colombiana de Psicología & C [4] C [4] & $10[2]$ & $9[4]$ \\
Avances en Psicología Latinoamericana & - C [4] & $10[2]$ & $16[1]$ \\
\hline
\end{tabular}

Las publicaciones que se encuentran en las posiciones más elevadas en CIRC, MIAR, Publindex y $\mathrm{H} 5$ son las que están presentes en las bases de datos de Scopus y WoS, siendo la excepción la revista Avances en Psicología Latinoamericana, rotativo que hasta septiembre del 2017 estaba escalafonado en Publindex como A1 y que a finales del año 2018 no estaba valorado en esta plataforma. De la misma manera, todas las correlaciones entre las cuatro mediciones son positivas (Tabla 3), aunque únicamente son significativas las correlaciones existentes entre las puntuaciones de Publindex H5 de Google $(r=0.863 ; p<0.01)$, MIAR - H5 de Google $(r=0.682 ; p<0.05)$ y CIRC - MIAR $(r=0.63 ; p<0.05)$.

\section{Tabla 3}

Correlaciones de Spearman de las diferentes clasificaciones

\begin{tabular}{lrrrr}
\hline Clasificaciones & \multicolumn{1}{c}{ Publindex } & CIRC & MIAR & H5 \\
\hline Publindex & 1 & & & \\
CIRC & $0.08(p=0.78)$ & 1 & & \\
MIAR & $0.5(p=0.13)$ & $0.63(p<0.05)$ & 1 & \\
H5 & $0.86(p<0.01)$ & $0.35(p=0.26)$ & $0.68(p=0.03)$ & 1 \\
\hline
\end{tabular}

Es llamativo que las dos bases de datos de procedencia ibérica (CIRC y MIAR) coincidan en sus planeamientos bibliométricos de evaluación sobre la calidad y prestigio de las revistas de psicología colombianas valoradas en Publindex, mientras que las discrepancias entre el índice del Ministerio de Educación de Colombia y estas dos matrices de datos españolas son más que notables, en especial con el CIRC, el único sistema que no obtuvo correlaciones significativas con el índice $\mathrm{H} 5$ de Google. De las discordancias encontradas entre las puntuaciones de Publindex y CIRC, se puede indicar que las puntuaciones son inferiores en esta plataforma bibliométrica, siendo el caso de la revista Avances en Psicología Latinoamericana nuevamente excepcional, pues a pesar de estar indexada en Scopus, se mantendría en la categoría C de CIRC. Esquemáticamente, podemos clasificar las puntuaciones de las revistas colombianas de psicología de la siguiente manera:

Valoraciones más positivas en Publindex que en CIRC: ocho revistas: Acta Colombiana de Psicología, Diversitas: Perspectivas en Psicología, Pensamiento Psicológico, Psicología desde el Caribe, Psychologia: Avances de la disciplina, Revista CES Psicología, Suma Psicológica e Informes Psicológicos.

Valoraciones más positivas en CIRC que en Publindex: cero revistas.

Valoraciones similares Publindex-CIRC: cinco revistas: Revista Latinoamericana de Psicología, Universitas Psychologica, Pensando Psicología, Psicogente y Revista Colombiana de Psicología.

Similares apuntes al equiparar las puntuaciones de CIRC con los cuartiles sacados del índice H5 de Google [para prevenir 
deformaciones, se re-evaluaron los cuartiles de CIRC (A2: 1 / B: 2 / C: 3 / D: 4)], pues siete manuscritos tienen mejores estimaciones en $\mathrm{H} 5$ en contraste con dos publicaciones que alcanzan una mayor evaluación en CIRC (Revista Colombiana de Psicología y Psicogente) y tres que obtienen puntuaciones parejas (Revista Latinoamericana de Psicología, Acta Colombiana de Psicología e Informes Psicológicos).

Menores discrepancias hay entre Publindex y MIAR, pues entre ambos indicadores bibliométricos existen mayores coincidencias a la hora de catalogar documentos en un mismo rango que la anterior plataforma, y solo hay dos casos de escritos que varíen tan drásticamente entre los dos ránquines (si se transforma antes el índice MIAR en puntuaciones cuartiles: las revistas Universitas Psychologica y Revista Colombiana de Psicología). A modo esquemático, las desemejanzas entre Publindex y MIAR se pueden sintetizar del siguiente modo [para evitar distorsiones en la medición, se reevaluaron los cuartiles de Publindex (A2: 1 / B: 2 / C: 3) y se clasificaron las puntuaciones MIAR en tres rangos]:

Valoraciones más positivas en Publindex que en MIAR: tres revistas: Pensamiento Psicológico, Psicología desde el Caribe y Suma Psicológica.

Valoraciones más positivas en MIAR que en Publindex: tres revistas: Universitas Psychologica, Pensando Psicología y Revista Colombiana de Psicología.

Valoraciones similares Publindex-MIAR: cuatro revistas: Revista Latinoamericana de Psicología, Acta Colombiana de Psicología, Informes Psicológicos y Psicogente.

Estas disparidades se hacen más notables si se comparan las puntuaciones de Publindex con el índice $\mathrm{H} 5$ de Google. Se reevaluaron los cuartiles de Publindex de igual forma que en MIAR y se codificaron tres niveles de valoraciones del índice $\mathrm{H} 5$, obteniéndose los siguientes resultados:

Valoraciones más positivas en Publindex que en $\mathrm{H} 5$ : cero revistas.

Valoraciones más positivas en $\mathrm{H} 5$ que en Publindex: dos revistas: Psicología desde el Caribe y Universitas Psychologica.
Valoraciones similares Publindex-H5: nueve revistas.

\section{Discusión}

La baja correlación obtenida por la matriz CIRC con el índice H5 de Google y el sesgo evaluativo, encontrado en la medición de las revistas de psicología de Colombia en esta base de datos, en comparación con otras similares herramientas como MIAR, son indicios relevantes que podrían afirmar que, como instrumento predictivo de la calidad y visibilidad de textos científicos, CIRC podría presentar algunas carencias. Por consiguiente, se puede concluir, en definitiva, que existe en CIRC una clara tendencia a minusvalorar la naturaleza de las revistas de psicología de Colombia que no se presenta en otros índices, teniendo que rechazarse de plano la justificación de que el índice utilizado en esta nación sudamericana (Publindex) es más laxo que el sistema CIRC, pues hasta septiembre del año 2017 había 28 revistas científicas de Colombia en la categoría A1 de Publindex, de las cuales ocho eran de psicología (Machuca, 2017; Ossa \& Cudina, 2016), mientras que en el año 2018, solo existía una única revista en la máxima posición y era ajena a la temática psicológica (Análisis Político).

La confrontación H5-Publindex-CIRC también evidencia que Publindex es más estricto con los propios escritos colombianos de psicología que otros mecanismos de origen extranjero. Complementario a lo anterior, es evidente que la reiterada crítica que afirma que Publindex castiga a las revistas de América Latina y privilegia a los indicadores anglosajones o europeos (Valencia et al., 2017) no es una simple queja autojustificativa acerca de las limitaciones existentes en recursos humanos y económicos de los investigadores e instituciones de Colombia (Ossa \& Cudina, 2016; Restrepo-Ochoa, 2017), teniendo ese lamento global un sustento empírico.

Lo dicho concordaría con las recomendaciones dadas por Aguillo-Caño et al. (2017) a los estamentos públicos de Colombia que rigen todo lo relacionado con el ámbito científico, al señalar 
que se debe seleccionar de forma cuidadosa las bases de datos que nutren las clasificaciones que se manejan para así obtener indicadores relativos por cada disciplina más fiables.

Es por ello por lo que, aunque las revistas de psicología de América Latina (y en particular las colombianas) han mejorado de manera continua su proceso editorial respecto a épocas anteriores (López-López, 2018; López-López et al., 2010), las dinámicas político-económicas que dirigen la investigación son las que determinan cómo se regula y difunde el conocimiento científico y a veces esta influencia es la que provoca condiciones nocivas para la ciencia (LópezLópez, 2018). Véanse como ejemplo, las métricas de las revistas de psicología que se han revisado en este documento, y compárense con los excelsos indicadores obtenidos en el año 2018 por otras publicaciones de origen venezolano que tan solo dos años más tarde fueron retiradas de la base de datos Scopus.

De este modo, uno de los aspectos más negativos que ha producido esta especialización es que los investigadores, en menor o mayor medida, han modificado sus metas profesionales, pues ahora publicar en los supuestos medios reconocidos como de alta rigurosidad es uno de los fines últimos (y no el medio), y parece que la valía y méritos de los trabajos en sí tienen una importancia menor. Esta actitud se ve reflejada en la conducta de los investigadores nóveles al cambiar este colectivo la manera de difundir sus investigaciones y trazar sus intereses laborales únicamente por los criterios de las agencias evaluadoras (Masip, 2011).

La obsesión mercantilista de los creadores en conseguir que sus investigaciones estén catalogadas en las revistas más reconocidas e insignes junto a la perpetua inquietud de los directores de las publicaciones en las mediciones de calidad (Ordorika, 2018), no nos debe impedir recordar que una revista es un transmisor central la información científica en la que los autores deben intentar alcanzar la excelencia y obrar siempre de manera recta, a pesar de todos los inconvenientes, en contraste con las malas artes de las denominadas revistas depredadoras (Somoza-Fernández et al., 2016). Por lo tanto, lograr índices bibliométricos elevados y mejorar los ya existentes (como CIRC o Publindex) es una buena señal que apunta a que el autor o la revista se dirige hacia la dirección correcta pero nunca deberá ser su meta final.

Así pues, consideramos que el vigente panorama académico global asume de manera errónea que los lectores, autores, editores, evaluadores de comunicaciones científicas no están interesados en el saber y en la erudición, al convertirse respectivamente en clientes, productores, distribuidores y auditores (Restrepo-Ochoa, 2017), y esto es algo que debe ser subsanado. Como bien indican Gorraiz y Jiménez-Contreras (2018), en un ensayo humorístico que analiza los divergentes intereses de los actores involucrados en la revelación de este tipo de textos (p. 440):

Lo que está claro es que la ciencia se está comercializando cada vez más. El científico se está convirtiendo en una especie de ejecutivo, con un montón de tareas que le apartan de su trabajo y le impiden desarrollar una investigación seria. Y según parece estamos obligados a producir y publicar cada vez más y más. Y lo que es peor, es que ya no se nos pide sólo que publiquemos, sino también que promocionemos nuestras publicaciones.

Es por ello por lo que planteamos, al igual que opina Ordorika (2018), una defensa de la investigación local basada en los idiomas originales de los autores, pero manteniendo siempre los niveles de calidad de los estudios presentados, requiriéndose para ello inversiones para mejorar tanto los recursos físicos como los humanos (Jarama-Barrios et al., 2012). En relación con la férrea dependencia que sufrimos los investigadores hacia los indicadores bibliométricos, léanse nuestras críticas como posibilidad de mejorar y no como ataques hacia la ardua labor de otros compañeros; así planteamos la posibilidad de diseñar indexaciones específicas por cada campo de conocimiento humano, en los que se valoren variables cualitativas (López-López, 2017), si bien creemos que lo fundamental en la actividad científica siempre será el contenido de nuestros textos, el debate 
abierto de ideas y la autorregulación de cada persona.

Las directrices de indexación por las que se rige la producción científica de un área del conocimiento como la psicología en Colombia no es un aspecto baladí ni trivial. Aunque nunca se puede satisfacer a todos los investigadores si es plausible hacer modificaciones pertinentes basadas en la ciencia para así conseguir que las mejoras en los indicadores tengan un calado a largo plazo, en contraste a los continuos y bruscos bandazos políticos realizados por la antigua Colciencias (Aguillo-Caño et al., 2017).

Dentro de las limitaciones existentes de esta investigación, se debe recalcar que este trabajo tiene un alcance exploratorio, por lo que si bien los hallazgos encontrados revelan diferencias evidentes entre dos matrices de datos de origen ibérico (CIRC y MIAR) que reúnen información para la identificación de las principales revistas de psicología colombianas, el carácter intrínseco de este estudio imposibilita generalizar los descubrimientos encontrados, siendo una de las principales restricciones el número de datos recogidos. Por lo tanto, en estudios futuros recomendamos incorporar información de las matrices con las que se ha trabajado y otras (Serrate \& Rovira, 2017) pero con revistas de áreas temáticas más amplias (ciencias de la salud y ciencias sociales de Colombia) para así poder confirmar nuestros resultados desde una perspectiva más global.

\section{Referencias}

Aguillo-Caño, I., Uribe-Tirado, A., \& López-López, W. (2017). Visibilidad de los investigadores colombianos según sus indicadores en Google Scholar y ResearchGate. Diferencias y similitudes con la clasificación oficial del sistema nacional de ciencia - COLCIENCIAS. Revista Interamericana de Bibliotecología, 40(3), 221-230. https://doi.org/10.17533/u dea.rib.v40n3a03

Ávila-Toscano, J. H., \& Marenco-Escuderos, A. D. (2016). Producción bibliométrica y redes de cooperación en la Revista Psicología desde el Caribe. Psicología desde el Caribe, 33(1), 66-80. https://doi.org/10.14482/psdc .33 .1 .8063

Bar-Ilan, J. (2008). Which h-index? A comparison of WoS, Scopus and Google Scholar. Scientometrics, 74(2), 257-271. htt ps://doi.org/10.1007/s11192-008-0216-y

Charum, J. (2004). La construcción de un sistema nacional de indexación, el caso de publindex. Convergencia, 35, 293-309. http s://www.redalyc.org/pdf/105/10503511.pdf

Delgado-López, E., Orduña-Malea, E., JiménezContreras, E., \& Ruiz-Pérez, R. (2014). H Index Scholar: el índice h de los profesores de las universidades públicas españolas en humanidades y ciencias sociales. El profesional de la Información, 23(1), 87-94. h ttps://doi.org/10.3145/epi.2014.ene.11

Díaz, G. M. (2017). Incertidumbre ante la implementación del nuevo modelo de medición de revistas científicas en Colombia. Revista TecnoLógicas, 20(38), 9-13. https://doi.org/10.22430/22565337.5 79

Díaz, Y., Baena, M. A., \& Baena, G. R. (2017). MOOC en la educación: un acercamiento al estado de conocimiento en Iberoamérica. Revista Iberoamericana para la Investigación y el Desarrollo Educativo, 8(15). https://doi.or g/10.23913/ride.v8i15.299

Espinosa, N., \& Insuasty, A. (2015). El conocimiento inventariado. Apuntes críticos sobre el modelo de indexación de las publicaciones académicas en Colombia. El Ágora USB, 15(1), 95-113. https://doi.or $\mathrm{g} / 10.21500 / 16578031.4$

Fernández-Abad, F. J. (2006). Evolución histórica de la función social de las bibliotecas públicas. Revista General de Información y Documentación, 16(2), 93-110. https://revistas.ucm.es/index.php/ RGID/article/view/RGID0606220093A

Gómez-Marín, J. E., \& Palacios, M. (2018). Una nueva etapa de Publindex: dolores de crecimiento. Revista Infectio, 22(1), 7-8. htt ps://doi.org/10.22354/in.v0i0.697 
Gorraiz, J., \& Jiménez-Contreras, E. (2018). Seis indicadores en busca de un sentido. El Profesional de la Información, 27(2), 432-440. https://doi.org/10.3145/epi.2018. mar. 22

Hirsch, J. E. (2005). An index to quantify an individual's scientific research output. Proceedings of the National Academy of Sciences of the United States of America, 102 (46), 16569-16572. PNAS.

Jarama-Barrios, B., Guerrero-Castro, J., GómezMorales, Y. J., \& López-López, W. (2012). Bibliometría e historia de las prácticas académicas locales: un esbozo a partir del caso de la psicología en Colombia. Avances en Psicología Latinoamericana, 29(2), 354-369. https://revistas.urosario.ed u.co/index.php/apl/article/view/2008/1744

López, M. (agosto, 2007). La cibermetría, una nueva alternativa para evaluar la visibilidad de la publicación académica electrónica. Razón y Palabra, 58. https://www.redalyc.or g/comocitar.oa?id=199520717015

López, L. E., \& Yung, I. (1998). Sobre las huellas de la voz: sociolingüística de la oralidad y la escritura en su relación con la educación. Ediciones Morata.

López-López, W. (2017). ¿Puede la cienciometría contribuir a la evaluación de la producción intelectual? Universitas Psichologica, 16(4), 1-3. https://revistas.javeriana.edu.co/index. php/revPsycho/article/view/20814

López-López, W. (2018). Las revistas de psicología de Iberoamérica: historia, estado y perspectivas. Universitas Psychologica, 17(3), 1-3. https://revistas.javeriana.edu.co /index.php/revPsycho/article/view/22424

López-López, W., García-Cepero, M. C., AguilarBustamante, M. C., \& Aguado-López, E. (2010). Panorama general de la producción académica en la psicología colombiana indexada en psicoredalyc, 2005-2007. Acta Colombiana de Psicología, 13(2), 35-46. https://www.redalyc.org/pdf/798/79 819279004.pdf

Machuca, F. (2017). Futuro de las publicaciones científicas en Colombia [Editorial]. Revista
Ingeniería y Competitividad, 19(2). https://d oi.org/10.25100/iyc.v19i2.5923

Martín-Martín, A., Orduña-Malea, E., \& Delgado-López, E. (2018). Coverage of highly-cited documents in Google Scholar, Web of Science, and Scopus: A multidisciplinary comparison. Scientometrics, 116(3), 2175-2188. https://d oi.org/10.1007/s11192-018-2820-9

Mas-Bleda, A., \& Aguillo, I. F. (2015). La web social como nuevo medio de comunicación y evaluación científica. UOC.

Masip, P. (2011). Efecto Aneca: producción española en comunicación en el Social science citation Index. Anuario ThinkEPI, 5, 206-210. http://eprints.rclis.org/15944/1/A nuario-ThinkEPI-2011-206-210-Masip.pdf

Mederos-Martin, A., \& Ruiz-Cabrero, L. (2001). Los inicios de la escritura en la Península Ibérica. Grafitos en cerámicas del bronce final III y fenicias. Complutum, 12, 97-112. https://www.academia.edu/113631 5/Los_inicios_de_la_escritura_en_la_Pen \%C3\%ADnsula_Ib\%C3\%A9rica_Grafitos _en_cer\%C3\% $\bar{A} 1$ micas_del_Bronce_Final III_y_fenicias

Mendoza, S., \& Paravic, T. (2006). Origen, clasificación y desafíos de las Revistas Científicas. Investigación y Postgrado, 21(1), 49-75. https://www.redalyc.org/pdf/658/65 821103.pdf

Moed, H. F. (2005). Citation analysis in research evaluation. Springer.

Ordorika, I. (2018). Las trampas de las publicaciones académicas. Revista Española de Pedagogía, 76(271), 463-480. https://doi. org/10.22550/REP-3-2018-04

Orduña-Malea, E., Martín-Martín, A., \& Delgado-López, E. (2016). Researchgate como fuente de evaluación científica: desvelando sus aplicaciones bibliométricas. El Profesional de la Información, 25(2), 303-310. http://profesionaldelainformacion .com/contenidos/2016/mar/18.pdf

Osca-Lluch, J., Veyrat, A., \& Morales, J. (2013). El consumo de información en 
humanidades. Arbor, 189(760). https://doi. org/10.3989/arbor.2013.760n2012

Ossa, J. C., \& Cudina, J. N. (2016). Sesenta años de las revistas de psicología en Colombia. Revista Guillermo de Ockham, 14(2), 7-17. h ttps://doi.org/10.21500/22563202.2720

Pérez-Anaya, O. (2017). Índice de Osk: una nueva medición bibliométrica para las revistas científicas. Revista Española de Documentación Científica, 40 (2). https://doi .org/10.3989/redc.2017.2.1418

Restrepo-Ochoa, D. A. (2017). Revista CES Psicología: una década de logros y desafíos en un contexto de capitalismo académico y cognitivo. Revista CES Psicología, 10(2), 1-2. https://www.redalyc.org/pdf/4235/423 553242011.pdf

Rodríguez, E., Naranjo, S., \& González, D. L. (2015). Publindex: más que un proceso de indexación. El Ágora USB. Revista de Ciencias Sociales, 15(1), 19-25. https://doi.o $\mathrm{rg} / 10.21500 / 16578031.1$

Rodríguez-Gairín, J. M., Somoza-Fernández, M., \& Urbano, C. (2011). MIAR: hacia un entorno colaborativo de editores, autores y evaluadores de revistas. El profesional de la información, 20(5), 589-595. https://doi.org /10.3145/epi.2011.sep.15

Rueda-Clausen, C. F., Villa-Roel, C., \& Rueda-Clausen, C. E. (2005). Indicadores bibliométricos: origen, aplicación, contradicción y nuevas propuestas. MedUNAB, 8(1), 29-36.

Ruiz-Corbella, M., Galán, A., \& Diestro, A. (2014). Las revistas científicas de Educación en España: evolución y perspectivas de futuro. Revista Electrónica de Investigación E̊ Evaluación Educativa, 20(2). https://doi.org/10.7203/relieve.20.2.4361

Salas, G., Ravelo-Contreras, E. L., Mejía, S., Andrades, R., Acuña, E., Espinoza, F., Núñez, M., Barboza-Palomino, M., Ventura-León, J., Caycho-Rodríguez, T., \& Pérez-Acosta, A. M. (2018). Dos décadas de Acta Colombiana de Psicología: un análisis bibliométrico. Acta Colombiana de Psicología, 21 (2), 13-25. https://doi.org/10.1 4718/ACP.2018.21.2.2
Salazar, M. (Ed.). (2013). Colciencias cuarenta años: entre la legitimidad, la normatividad y la práctica. Observatorio de Ciencia y Tecnología, Universidad Nacional de Colombia, Universidad del Rosario.

Schiltz, M. (2018). Science without publication paywalls: cOAlition $S$ for the Realization of Full and Immediate Open Access. PLoS Medicine, 15(9), 1-4. https://doi.org/10.137 1/journal.pmed.1002663

Serrate, L., \& Rovira, L. (2017). La base de datos Carhus Plust: una clasificación integrada por índices de citación y de calidad editorial para la evaluación de la actividad investigadora. El caso del Derecho. Revista de Educación y Derecho, 16. https://www.ra co.cat/index.php/RED/article/view/328825

Silva-Ayçaguer, L. C. (2012). El índice$\mathrm{H}$ y Google Académico: una simbiosis cienciométrica inclusiva. Revista Cubana de Información en Ciencias de la Salud, 23(2), 308-322. https://www.medigraphic.com/cgi -bin/new/resumen.cgi?IDARTICULO=37 253

Somoza-Fernández, M., Guallar, J., RodríguezGairín, J. M., \& Abadal, E. (2017). Presencia de revistas españolas en bases de datos internacionales. En E. Abadal (Ed.), Revistas científicas: situación actual y retos de futuro (pp. 161-178). Universidad de Barcelona.

Somoza-Fernández, M., Rodríguez-Gairín, J. M., \& Urbano, C. (2016). Presence of alleged predatory journals in bibliographic databases: analysis of Beall's list. El profesional de la Información, 25(5), 730-737. https://doi.org/10.3145/epi.2016.s ep.03

Torres-Salinas, D., Bordons, M., GiménezToledo, E., Delgado-López, E., JiménezContreras, E., \& Sanz-Casado, E. (2010). Clasificación integrada de revistas científicas (CIRC): propuesta de categorización de las revistas en ciencias sociales y humanas. El profesional de la Información, 19(6), 675-683. https://doi.org /10.3145/epi.2010.nov.15 
Torres-Salinas, D., Jiménez-Contreras, E., \& Robinson-García, N. (2014). Tendencias en mapas de la ciencia: co-uso de información científica como reflejo de los intereses de los investigadores. El profesional de la información, 23(3), 253-258. https://doi.org /10.3145/epi.2014.may.05

Urbano, C. (junio, 2003). Avaluació de revistes i avaluació de la recerca en humanitats i ciències socials a Catalunya: aproximació a un problema. BiD: Textos Universitaris de Biblioteconomia i Documentació, 10. http://b id.ub.edu/10urbano.htm

Valencia, J. F., Gelacio, J.D., \& Vanegas, H. I. (2017). Los modelos de indexación de revistas y la complementariedad con la investigación. Revista Ratio Juris, 12(24), 17-26. https://doi.org/10.24142/raju.v12n2 $4 \mathrm{a} 1$

Villalobos-Galvis, F., \& Puertas-Campanario, R. (2007). Impacto e internacionalidad de tres revistas iberoamericanas en revistas de psicología de España. Revista Latinoamericana de Psicología, 39(3), 593-608. http://www.scielo.org.co/pdf/rlps/ v39n3/v39n3a11.pdf

Villegas-Gallego, M. (2013). Nuevo modelo de indexación y homologación Publindex: apostándole cada vez más a la visibilidad internacional [Editorial]. Psicología desde el Caribe, 30(3), 7-8. http://rcientificas.uninorte.edu.co/ind ex.php/psicologia/issue/view/334

\section{Notas}

* Artículo de investigación. 